\title{
Predação oportunística de Molossus molossus (Pallas, 1766) (Chiroptera: Molossidae) por Rhinella jimi (Stevaux, 2002) (Anura: Bufonidae) na Caatinga, Pernambuco, Brasil
}

\author{
Luiz Augustinho Menezes da Silva ${ }^{1 *}$ \\ Ednilza Maranhão dos Santos ${ }^{2}$ \\ Fabiana Oliveira de Amorim ${ }^{3}$ \\ ${ }^{1}$ Universidade Federal de Pernambuco, Centro Acadêmico de Vitória, Núcleo de Biologia \\ Rua do Alto do Reservatório, s/n, CEP 55608-680, Vitória de Santo Antão - PE, Brasil \\ ${ }^{2}$ Universidade Federal Rural de Pernambuco, Unidade Acadêmica de Serra Talhada \\ ${ }^{3}$ Laboratório de Ecofisiologia e Comportamento Animal (LECA) \\ Universidade Federal Rural de Pernambuco \\ *Autor para correspondência \\ lamsilva@elogica.com.br
}

Submetido em 12/10/2009

Aceito para publicação em 23/02/2010

\section{Resumo}

A presente comunicação relata um evento de predação oportunística do morcego Molossus molossus pelo sapo Rhinella jimi, ocorrido em 29 de maio de 2003 na Caatinga, município de Orocó, Estado de Pernambuco, nordeste do Brasil.

Unítermos: anfíbio anuro, dieta, morcego, nordeste brasileiro

\section{Abstract}

Predation of Molossus molossus (Pallas, 1766) (Chiroptera: Molossidae) by Rhinella jimi (Stevaux, 2002) (Anura: Bufonidae) in the Caatinga, Pernambuco, Brazil. This communication is to report, through a fortuitous event, the predation of the bat Molossus molossus by the toad Rhinella jimi, on 29 May 2003 in the Caatinga, municipality of Orocó, state of Pernambuco, northeast Brazil.

Key words: amphibian anuran, bats, diet, northeast Brazil

Como predadores de morcegos podemos incluir vertebrados e invertebrados (Gillette e Kimbrough, 1970; Hermanson e Wilkins, 1986; Molinari et al., 2005), entre os vertebrados encontram-se, principalmente, registros de serpentes (e.g. Rodriguez-Duran, 1996; Esbérard e Vrcibradic, 2007), aves de rapina (Vernier, 1994; Escarlate-Tavares e Pessôa, 2005), mamíferos (e.g. Souza et al., 1997; Fellers, 2000; Bordignon, 2005) e poucos relatos para anfíbios (Esbérard et al., 2006; Gouveia et al., 2009).

O morcego Molossus molossus (Pallas, 1766) pertencente a família Molossidae é um insetívoro de ampla distribuição pela América do Norte, América 
Central e América do Sul (Simmons, 2005). No Brasil, é registrado em todas as regiões (Fábian e Gregorin, 2007), tanto em áreas urbanas (onde costuma ser abundante) quanto rurais, ocupando construções humanas e ocos de árvores, respectivamente (Bredt et al., 1998; Fabian e Gregorin, 2007). Na Caatinga é considerado comum e bem distribuído (Oliveira et al., 2003), havendo inclusive relatos de capturas em construções de áreas rurais (Willig, 1983). O comprimento do antebraço desses animais pode variar de 38 a 42mm (Husson 1962; Barquez et al., 1999) e a massa de 10 a $30 \mathrm{~g}$ (Bredt et al., 1998). Devido à morfologia alar dos molossídeos, estes animais quando caem no chão apresentam dificuldade em alçar vôo diretamente do solo, sendo necessário escalar alguma superfície para de lá se lançar em vôo livre (Freeman, 1981; Canals et al., 2001). Dessa forma, tornam-se presas fáceis para predadores de solo principalmente os oportunistas.

A maioria dos anfíbios anuros é generalista e é considerada forrageadora oportunista, sendo a disponibilidade de presas o fator predominante na determinação da dieta, aliada às limitações mecânicas dadas pelo tamanho relativo da presa/predador (Toft, 1981; Duellman e Trueb, 1994; Pough et al., 1998). Anuros alimentam-se de uma variedade de presas entre invertebrados e pequenos vertebrados, todavia relatos de espécies alimentando-se de mamíferos são raros (Duellman e Trueb, 1994; Esbérard et al., 2006; Gouveia et al., 2009). Neste grupo as espécies pertencentes ao gênero Rhinella (família Bufonidae) são conhecidas pela sua voracidade, apresentando uma dieta especialmente insetívora, podendo também se alimentar de invertebrados e pequenos vertebrados (Peña et al., 1996; Gouveia et al., 2009).

Rhinella jimi (Stevaux, 2002) é um anuro conhecido na região do semi-árido como sapo cururu ou sapo-boi, que possui ampla distribuição no Nordeste do Brasil principalmente na Caatinga e ocupa áreas próximas à habitação humana, lagoas, poças, margens de riachos, estradas e rodovias (Borges-Nojosa e Santos, 2005). Possui hábito noturno, modo reprodutivo tipo "1" (ovos e girinos exotróficos em ambientes lênticos), reprodução explosiva e alimentação composta especialmente por insetos; adotam geralmente uma estratégia de forrageador "senta e espera", podendo ser considerado como um animal generalista e oportunista (Moreira e Barreto, 1996). Recentemente foi relatado que $R$. jimi alimentou-se de morcego, um Pteronotus personatus (Wagner, 1843), família Mormoopidae em uma caverna no município de Itabaiana, Estado de Sergipe (Gouveia et al., 2009). Com o objetivo de contribuir para o conhecimento dessas espécies, a presente comunicação científica relata um evento de predação oportunística do morcego M. molossus por $R$. jimi em uma área de Caatinga, Estado de Pernambuco, nordeste do Brasil.

$\mathrm{O}$ evento de predação foi observado em uma área de reserva legal da CHESF (Companhia Hidro Elétrica do São Francisco), denominada Brígida ( $8^{\circ} 29^{\prime} 38^{\prime \prime}$, 39³2'48”W), no município de Orocó, Estado de Pernambuco, durante coletas de dados para o inventário e monitoramento da mastofauna e herpetofauna local. A região está localizada no sertão do médio São Francisco e fica a cerca de $520 \mathrm{~km}$ da cidade do Recife, essa área possui em seu entorno diversas culturas como plantação de banana e mamão além de uma Caatinga arbustiva com alguns elementos arbóreos.

O evento ocorreu durante uma sessão de capturas dos morcegos, com rede de neblina, na noite do dia 29 de maio de 2003 (temperatura do ar $24^{\circ} \mathrm{C}$ ). Os morcegos encontravam-se abrigados em uma edificação, entre o telhado e o forro, que era revestido internamente por gesso. Observou-se que os animais tinham como rotas de acesso ao interior do abrigo o espaço entre a parede e o telhado, nos diversos lados da casa. À cerca de 5m, diversos exemplares de Rhinella jimi (de diferentes classes etárias) forrageavam sob um poste de luz que atraia os insetos e estes ao pousarem no chão eram predados pelos animais.

Por volta das $17 \mathrm{~h} 50 \mathrm{~min}$, um morcego bateu na rede e caiu no chão apresentando dificuldade de voar; enquanto se debatia, um adulto de Rhinellajimi (CRC $135,7 \mathrm{~mm}$ e massa $-310 \mathrm{~g}$ ) se aproximou e o abocanhou no sentido antero-posterior, ingerindo-o velozmente, não sendo possível assim obter dados sobre a presa ingerida (sexo, peso e classe etária).

O sapo foi capturado, ainda com o morcego na boca para o registro fotográfico (Figura 1A), mesmo havendo 
essa interferência o anuro não liberou o morcego engolindo-o rapidamente (Figura 1B). As medidas de CRC e massa foram aferidas apenas do anuro, logo depois da ingestão, e o mesmo foi liberado em seguida no local do evento. As imagens foram depositadas na Coleção Herpetológica da Universidade Federal Rural de Pernambuco (arquivo - Predação 29032003).

Registros oportunísticos de predação de morcegos por vertebrados têm sido obtidos principalmente para gatos domésticos. Estes animais comportam-se como excelentes predadores de morcegos capturando-os em diferentes hábitats, ao caírem no chão ou ao passarem próximo desses predadores durante as atividades de forrageio e saída dos abrigos (Delpietro et al., 1987; Bredt et al., 2002). A predação oportunística durante os trabalhos de capturas de morcegos foi verificado por Oprea et al. (2006), quando o morcego Phyllostomus discolor consumiu um Glossophaga soricina na rede, assim como um evento de predação de morcegos por Didelphis albiventris (Gazarini et al., 2008), além de anfíbios anuros como $R$. jimi que consumiu Pteronotus personatus e registros de Leptodactylus vastus que foram observados alimentando-se de Natalus stramineus e Anoura caudifer (Esbérard et.al., 2006; Gouveia et al., 2009). Esberard et al. (2006) e Gouveia et al. (2009) abordam a necessidade de mais registros sobre predação de morcegos envolvendo anfíbios anuros para poder avaliar a importância desses eventos na natureza. Duellman e Trueb (1994) citam anuros predando pequenos roedores e outros anuros, todavia os autores destacam que esses eventos são raros na natureza. Apesar do caráter oportunista, essa comunicação registra pela primeira vez M. molossus como item alimentar de $R$. jimi.

\section{Agradecimentos}

Agradecemos a toda equipe técnica do projeto de fauna dos vertebrados de Itaparica em especial a Coordenadora Maria Adélia B. Oliveira.

\section{Referências}

Barquez, R. M.; Mares, M. A.; Braun, J. K. 1999. The bats of Argentina. Special Publications Museum of Texas Tech University, v.42, Ludbbock, USA, 275pp.

Borges-Nojosa, D.; Santos, E. M. 2005. Herpetofauna da área de Betânia e Floresta, Pernambuco. In: Araújo, F. S.; Rodal, M. J. N. \& Barbosa, M. R. V. (Eds). Análise das variações da biodiversidade do Bioma Caatinga - Suporte a estratégias regionais de conservação. Ministério do Meio Ambiente, Secretaria de Biodiversidade e Florestas, Brasília, Brasil, p.276-289.

Bordignon, M. O. 2005. Predação de morcegos por Chrotopterus auritus (Peters) (Mammalia: Chiroptera) no pantanal do Mato Grosso do Sul, Brasil. Revista Brasileira de Zoologia, 22 (4): 1207-1208.

Bredt, A.; Araujo, F. A. A.; Caetano Jr., J.; Rodrigues, M. G. R.; Yoshizawa, M.; Silva, M. M. S.; Harmani, N. M. S.; Massunaga, P. N. T.; Bürer, S. P.; Porto, V. A. R.; Uieda, W. 1998. Morcegos em áreas urbanas e rurais: Manual de manejo e controle. Fundação Nacional da Saúde, Brasilia, Brasil, 117pp.

Bredt, A.; Uieda, I.; Pinto, P. P. 2002. Visitas de morcegos fitófagos a Muntigia calabura L. (Muntigiaceae) em Brasília, Centro-Oeste do Brasil. Revista Brasileira de Zoociências, 4 (1): 111-122.

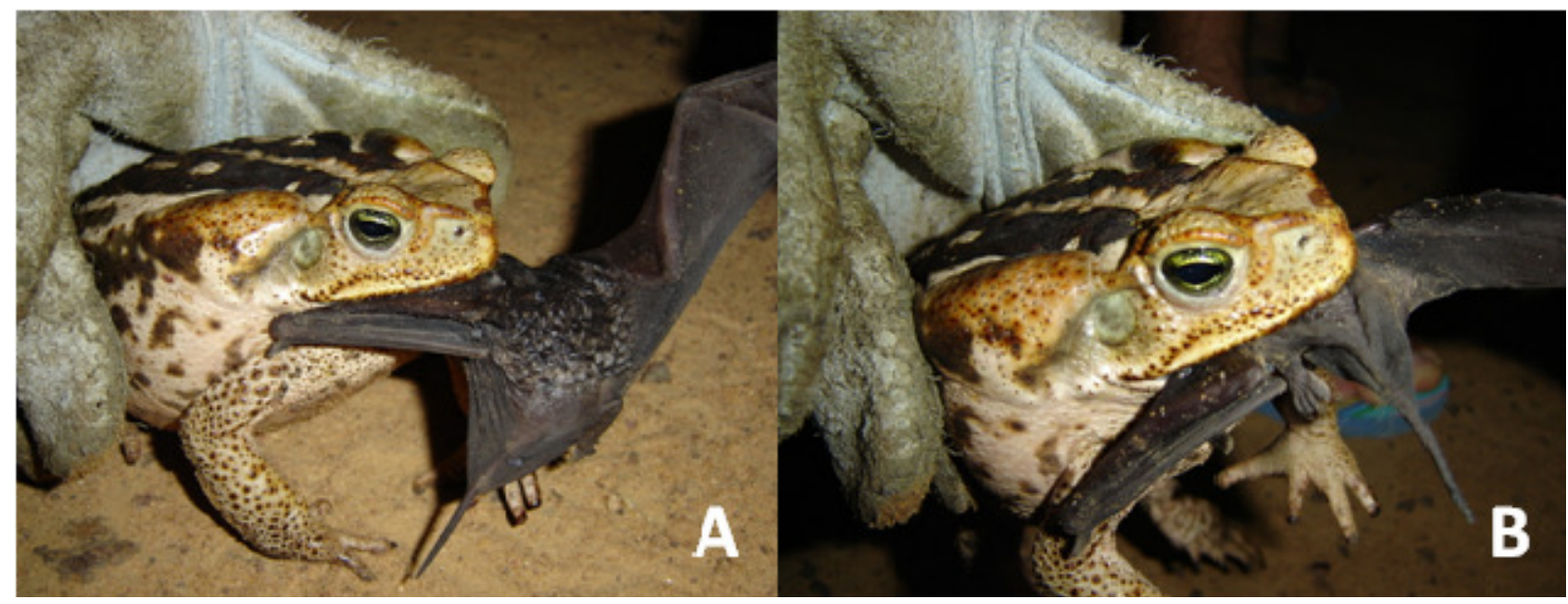

FIGURA 1: Rhinella jimi predando Molossus molossus em 29 de maio de 2003, no município de Orocó, Pernambuco, Brasil. A - Anuro abocanhando o morcego na porção antero-posterior; B - Finalização do processo de ingestão do morcego. 
Canals, M.; Iriarte-Diaz, J.; Olivares, R.; Novoa, F. F. 2001. Comparison of the wing morphology of Tadarida brasiliensis (Chiroptera: Molossidae) and Myotis chiloensis (Chiroptera: Vespertilionidae) as representatives of two flight patterns Revista Chilena de Historia Natural, 74 (3): 699-704.

Delpietro, H.; Fábregas, F.; Díaz, M. 1987. Riesgos de transmision rabica en la predacion de quiropteros por carnivoros domesticos. Veterinária Argentina, 4 (32): 119-124.

Duellman, W. E.; Trueb, L. 1994. Biology of amphibians. McGraw-fill Book Company, New Cork, USA, 670pp.

Esbérard, C. E. L.; Jordão, T.; Costa, L. M.; Bergallo, H. G. 2006. Leptodactylus labyrinthicus (labyrinthicus frog). Prey. Herpetological Review, 37 (2): 204.

Esbérard, C. E. L.; Vrcibradic, D. 2007. Snakes preying on bats: New records from Brazil and a review of recorded cases in the Neotropical Region. Revista Brasileira de Zoologia 24 (3): 949953.

Escarlate-Tavares, F.; Pessôa, L. M. 2005. Bats (Chiroptera, Mammalia) in barn owl (Tyto alba) pellets in northern pantanal, Mato Grosso, Brazil Mastozoología Neotropical, 12 (1): 61-67.

Fabian, M. E.; Gregorin, R 2007. Família Molossidae In: Reis, N. R. dos; Peracchi, A. L.; Pedro, W. A. \& Lima, I. P. (Eds). Morcegos do Brasil. Nélio R. dos Reis, Londrina, Brasil, p.149-165.

Fellers, G. M. 2000. Predation on Corynorhinus townsendii by Rattus rattus. The Southwestern Naturalist, 45 (4): 1-4.

Freeman, P. W. 1981. A multivariate study of the family Molossidae (Mammalia, Chiroptera): morphology, ecology, evolution. Fieldiana Zoology, 7: 1-43.

Gazarini, J.; Brito, J. E. C.; Bernardi, I. P. 2008. Predações oportunísticas de morcegos por Didelphis albiventris no sul do Brasil. Chiroptera Neotropical, 14 (2): 408-411.

Gillette, D. D.; Kimbrough, J. D. 1970. Chiropteran mortality. In: Slaughter, B. H. \& Walton, D. W. (Eds). About bats. Southern Methodist University Press, Dallas, USA, p.262-283.

Gouveia, S. F.; Rocha, P. A.; Milkalauskas, J. S.; Silveira, V. V. 2009. Rhinella jimi (Cururu toad) and Leptodactylus vastus (Northeastern pepper frog). Predation on bats. Herpetological Review, 40: 210.

Hermanson, J. W.; Wilkins, K. T. 1986. Preweaning mortality in a Florida maternity roost of Myotis austroriparius and Tadarida brasiliensis. Journal of Mammalogy, 67: 751-754.

Husson, A. M. 1962. The bats of Suriname. Zoologische Verhandelingen, 58: 1-282.

Kunz, T. H. 1982. Ecology of bats. Plenum Press, New York, USA, $425 \mathrm{pp}$.
Molinari, J.; Gutiérrez, E. E.; Ascenção, A. A.; Nassar, J. M.; Arends, A.; Márquez, R. J. 2005. Predation by giant centipedes, Scolopendra gigantea, on three species of bats in a Venezuelan cave. Caribbean Journal of Science, 41 (2): 340-346.

Moreira, G.; Barreto L. 1996. Alimentação e variação sazonal na frequência de capturas de anuros em duas localidades do Brasil Central. Revista Brasileira Zoologia, 13 (2) 313-320.

Oliveira, J. A. de; Gonçalves, P. R.; Bonvicino, C. R. 2003. Mamíferos da Caatinga. In: Leal, I. R.; Tabarelli, M. \& Silva, J. M. C. (Eds). Ecologia e Conservação da Caatinga. Universidade Federal de Pernambuco, Recife, Brasil, p.275-337.

Oprea, M.; Vieira, T. B.; Pimenta; V. T.; Mendes, P.; Brito, D.; Ditchfield, A. D.; Knegt, L.V. de; Esbérard, C. E. L. 2006. Bat predation by Phyllostomus hastatus. Chiroptera Neotropical, 12 (1): 255-258.

Peña, J. C.; Barrantes, R. B.; Ugalde, D. R. 1996. Hábitos alimentares de Bufo marinus (Anura: Bufonidade) en Costa Rica. Revista de Biologia Tropical, 19 (5): 702.

Peracchi, A. L.; Albuquerque, S. T. 1971. Lista provisória dos quirópteros dos Estados do Rio de Janeiro e Guanabara, Brasil (Mammalia, Chiroptera). Revista Brasileira de Biologia, 31 (3): 405-413.

Pough, F. H.; Handrews, R. M.; Cadle, J. E.; Crump, M. L.; Savitzky, A. H.; Wells, K. D. 1998. Herpetology. Prentice Hall, New Jersey, USA, 577pp.

Rodríguez-Durán, A. 1996. Foraging ecology of the Puerto Rican boa: Bat predation, carrion feeding, and piracy. Journal of Herpetology, 30: 533-536.

Simmons, N. B. 2005. Order Chiroptera. In: Wilson, D. E.; Reeder, D. M. (Eds.). Mammal Species of the World: a taxonomic and geographic reference. 3.ed. v.1. Baltimore: Johns Hopkins University Press, p. 312-529.

Souza, L. L.; Ferrari, S. F.; Pina, A. L. 1997. Feeding behaviour and predation of a bat by Saimiri sciureus in a semi-natural Amazonian environment. Folia Primatologica, 68: 194-198.

Toft, C. A. 1981. Feeding ecology of Panamanian litter anurans: patterns in diet and feeding mode. Journal of Herpetology, 15: 139-144.

Vernier, E. 1994. Predazione di chirolteri da parte del barbagianni (Tyto alba) in Italia. Hystrix, 5 (1-2): 105-107.

Willig, M. R. 1983. Composition, microgeographic variation, and sexual dimorphism in caatingas and cerrado bat communities from Northeast Brazil. Bulletin of Carnegie Museum of Natural History, 23: 1-131. 